\title{
Post-intubation tracheal stenoses: what is the curative yield of the interventional pulmonology procedures?
}

\author{
M. Patelli1, S. Gasparini2
}

Monaldi Arch Chest Dis 2007; 67: 2, 71-72.

1 Unit of Thoracic Endoscopy, Departement of Oncology; Maggiore-Bellaria Hospital; Azienda USL Bologna,

2 Pulmonary diseases Unit, Departement of Internal Medicine, Immunoallergic and Respiratory Diseases; Azienda Ospedaliera Universitaria "Ospedali Riuniti” Ancona, Italy.

Correspondence: Marco Patelli, Endoscopia Toracica-Pneumologia, Ospedale Maggiore, Largo Nigrisoli 2, 40100 Bologna, Italy; e-mail: marco.patelli@ausl.bologna.it

Focal non-neoplastic stenoses of the trachea are uncommon and many of these are complications of either endotracheal intubation or placement of tracheostomy tube. The pathogenesis of post-intubation tracheal stenoses (PITS) may be related to multiple factors (infection due to bacterial colonization, meccanical irritation, hypotension, gastroesophageal reflux, underlying illness), but the ischemia determined by the pressure of the endotracheal tube balloon against the tracheal wall plays the major role. It results in mucosal damage, ulceration, chondritis and leads to scarring and progressive stenosis $[1,2]$.

The incidence of iatrogenic airway injury have markedly decreased over the years with the use of endotracheal tubes with large volume-low pressure cuffs, but tracheal stenosis continues to be a complication of prolonged endotracheal intubation and tracheostomy.

The incidence of such a complication varies in the literature from $10 \%$ to $31 \%$, but stenoses greater than $50 \%$ of the tracheal diameter are $1-2 \%$ [3].

The stenoses may be:

1) web-like type, when the circumferential strictures of the trachea involves the mucosa for a short segment (maximum $1 \mathrm{~cm}$ in length), in some cases with inflammation, but without any damage of the cartilages;

2) pseudoglottic type, due to lateral fracture of cartilage consequent to tracheostomy;

3) complex type, when the strictures of the trachea is more than $1 \mathrm{~cm}$ long and is often associated with various degrees of cartilage involvement, malacia and inflammation [4].

Finally, according to their location, PITS can be distinguished in:

a) subglottic, in cases of too large tubes (frequent in womens) or in the case of the proximal anterior cricoid erosion by a high tracheostomy;

b) at the stoma site after tracheostomy;

c) at the site where the inflatable cuff rested;

d) between the stoma and the level of the cuff (tracheal malacia); and e) at the site where the tip of the tube may impinge on the tracheal wall [5].

The management of PITS is still not well defined and no controlled randomised studies have been performed to evaluate the role of open surgery versus the bronchoscopic treatment, including bronchoscopic or balloon dilatation, laser resection and stent placement. The surgical tracheal sleeve resection is considered the definitive treatment of choice with a failure rate ranging from $5 \%$ to $15 \%[6,7]$ and a post-operative mortality ranging from $1.8 \%$ to $5 \%$ [4]. However, several patients with PITS are in poor general conditions and are not suitable for surgery. Furthermore, there are cases with severe stenosis and consequent acute respiratory failure that require urgent bronchoscopic intervention before considering curative surgery.

Based on these considerations, in 1999 Brichet et al. proposed an algorithm for the management of PITS. This was validated by the analysis of results in a series of 32 consecutive patients [4]. An initial bronchoscopic treatment was performed in all patients. In cases with "web-like stenosis" a radial incision of the fibrotic membrane by laser followed by gentle mechanical dilatation with bronchoscope was performed.

This treatment was repeated up to three times in cases of recurrence, before considering surgery or stent placement for inoperable patients. The success rate of bronchoscopic treatment was $66 \%$. In cases with complex stenosis the initial treatment was bronchoscopic dilatation followed by silicone stent placement. Six months later, the stent was removed in patients suitable for surgery and in cases of recurrence a tracheal sleeve resection was performed. In inoperable patients, after six months the stent was left in place or removed, and placed again in case of recurrence.

The success rate of bronchoscopic treatment after stent removal in cases of complex stenosis was $17.6 \%$. The low definitive curative yield of bronchoscopic procedures in cases of complex stenosis and the reported risk that a stent might lengthen the tracheal segment to be resected [4], induces to revisit this algorithm. In cases of complex stenosis, if the patient is suitable for surgery, the tracheal sleeve re- 
section could be the first option or considered after bronchoscopic dilatation (without stent insertion) if there is need to overcome an urgent respiratory failure condition.

In April 2006, the Interventional Pulmonology Study Group of the Italian Association of Pulmonologists (AIPO) organised in Bologna a Consensus Conference on Interventional Pulmonology procedures, where the following recommendations about this argument were shared:

1) The endoscopic treatment is only available for those patients not suitable for surgery because of the excessive length of the stenosis or because of the presence severe comorbidities (Level of recommendation B).

2) The urgent endoscopic treatment of critical weblike or complex stenoses (diameter less than 5 $\mathrm{mm}$ ) is universally accepted, since it permits the acute phase to be overcome without performing more invasive and dangerous procedures, such as tracheotomy. (Level of recommendation B).

3) The endoscopic treatment is the gold standard for simple lesions, namely granulomas, fibrotic bridges or web-like stenoses, with a very high success rate. (Level of recommendation B).

4) When one or more cartilagineous rings are involved, the endoscopic treatment is normally contraindicated unless surgery is not feasible. (Level of Recommendation B).

A great contribution on this topic is published in this issue of Monaldi Archives for Chest Diseases, where Cavaliere and Coll. [8] report their experience on the bronchoscopic treatment of PITS and discuss the management of this pathological condition. The results obtained by the Authors are excellent with a success rate of bronchoscopic laser assisted tracheal dilatation for "web-like stenosis" of $92 \%$ without the use of stents. Also for complex stenosis, the paper shows a high rate of stable and good results with the bronchoscopic treatment, that allows a definitive and curative success to be obtained in 35 out of $60 \mathrm{pa}-$ tients $(58.3 \%$ ) after stent removal. In 25 cases $(41.6 \%)$ the bronchoscopic treatment was unable to obtain a definitive cure. Among these, thirteen non surgical patients are reported still to have the stent in place with a good quality of life and twelve patients (20\%) have been referred to surgeon for the tracheal resection and anastomosis.

What are the comments that can be made after reading this paper?

The first observation concerns the very high percentage of success in cases of complex stenosis. The difference from other papers is the timing of stent removal, that in the experience of Cavaliere et al. is delayed (11.6 \pm 4.6 months) in respect to the six months reported by Brichet et al. [4]. We can only speculate that this could be an important factor for the outcome, since there are no comparative studies that focus on the best length of time to keep in place a stent. Randomised trials in this sense would be desirable. The second important point that must be underlined in the paper of Cavaliere et al. is that in none of the treated patients the bronchoscopic treatment determined the need to extend the length of surgical resection over the original length of the stenosis. It must be emphasised that such excellent results have been obtained in a centre with a great experience and by operators that are highly skilled in therapeutical bronchoscopy. In this field, the operator experience plays a major role in determining the curative yield of the procedures, since a mistake on the technique or on the choice of the stent (wrong length or diameter or type of stent) or on the site of stent placement, may determine a failure of the treatment result. Moreover, the same considerations can be made for surgery, since also surgical treatment results are greatly influenced by the operator skill.

Finally, a consideration about the stents. They support the airways walls in cases of unstable stenoses after laser assisted mechanical dilatation. The stents have to be easy to remove and replace and the silicone Dumon stents are still the "gold standard" for the benign tracheobronchial stenoses [9]. The use of self-expanding silicone stent in this situation is associated with a high complication rate [10], while metallic stents may be difficult to remove.

In conclusion, the future success and increasing use of the bronchoscopic treatment of iatrogenic tracheal stenoses is likely to depend on the achievement of the following goals:

1) a considerable percentage of patients should be managed successfully;

2) the failure of the endoscopic treatment in patients who were potentially surgical candidates should not negatively affect the possibility and the success rate of subsequent open surgery.

The encouraging data obtained by Cavaliere and Coll. suggest that the above targets can be really reached. But these results also support the statement that this kind of pathology should be managed in referential centers with great experience in therapeutical bronchoscopy and with the availability of multidisciplinary competences.

\section{References}

1. Stark P. Imaging of tracheobronchial injuries. J Thoracic Imaging 1995; 10: 206-219.

2. Sue RD, Susanto I. Long-term complications of artificial airways. Clin Chest Med 2003; 24: 457-471.

3. Norwood S, Vallina S, Short K Saigusa M, Fernandes LG, McLarty JW. Incidence of tracheal stenosis and other late complications after percutaneous tracheostomy: Ann Surg 2000; 232 (2): 233-241.

4. Brichet A, Verkindre C, Dupont J, Marquette CH. Multidisciplinary approach to management of postintubation tracheal stenoses. Eur Respir J 1999; 13: 888-893.

5. Grillo HC. Surgery of the trachea and bronchi. BC Decker Inc. Hamilton, London 2004

6. Rea F, Callegaro D, Sartori F. Benign tracheal and laryngotracheal stenosis: surgical treatment and results. Eur J Cardiothorac Surg 2002; 22: 352-356.

7. Grillo HC, Donahue DM, Mathisen DJ. Postintubation tracheal stenosis. Treatment and results. J Thorac Cardiovasc Surg 1995; 109: 486-493.

8. Cavaliere S, Bezzi M, Toninelli C, Foccoli P. Management of post-intubation tracheal stenoses using the endoscopic approach. Monaldi Arch Chest Dis 2007; 67: 73-80.

9. Dumon JF. A dedicated tracheobronchial stent. Chest 1990; 97: 328-32.

10. Gildea TR, Murthy SC, Sahoo D, Mason DP, Mehta AC Performance of a self-expanding silicone stent in palliation of benign airway conditions. Chest 2006; 130: 1419-1423. 